Delft University of Technology

\title{
BDC-Decomposition for global influence analysis
}

Blanchini, Franco; Giordano, Giulia

DOI

10.1109/LCSYS.2018.2866903

Publication date

2019

Document Version

Accepted author manuscript

Published in

IEEE Control Systems Letters

\section{Citation (APA)}

Blanchini, F., \& Giordano, G. (2019). BDC-Decomposition for global influence analysis. IEEE Control Systems Letters, 3(2), 260-265. [8444706]. https://doi.org/10.1109/LCSYS.2018.2866903

\section{Important note}

To cite this publication, please use the final published version (if applicable).

Please check the document version above.

Other than for strictly personal use, it is not permitted to download, forward or distribute the text or part of it, without the consent of the author(s) and/or copyright holder(s), unless the work is under an open content license such as Creative Commons.

\section{Takedown policy}

Please contact us and provide details if you believe this document breaches copyrights.

We will remove access to the work immediately and investigate your claim. 


\title{
$B D C$-decomposition for global influence analysis
}

\author{
Franco Blanchini $^{a}$ and Giulia Giordano ${ }^{b}$
}

\begin{abstract}
In biochemical networks, the steady-state inputoutput influence is the sign of the output steady-state variation due to a persistent positive input perturbation; if the sign does not depend on the value of the strictly positive system parameters, the influence is structural. As recently shown for small perturbations, when the linearised system approximation is valid, steady-state input-output influences can be structurally assessed, for biochemical networks with $m$ unknown parameters, by means of a vertex algorithm with complexity $2^{m}$. This paper shows that the structural input-output influence of a biochemical network is a global property, which does not require any small-perturbation assumption. It also shows that, using a new algorithm, the complexity can be reduced down to $2^{m-n}$, where $n$ is the system order, thus drastically reducing the computation time. Finally, when the uncertain parameters belong to known intervals, non-conservative bounds are given for the steady-state ratio between output and input, allowing for sensitivity analysis.
\end{abstract}

Index Terms-Biomolecular systems, Network analysis and control, Systems biology

\section{INTRODUCTION}

W HEN modelling and analysing biomolecular systems with a mathematical approach, a big challenge is to deal with the huge uncertainties and variability in their parameters. Still, biological systems show an extraordinarily robust behaviour [1], [23]: crucial qualitative behaviours are always preserved, regardless of parameter values, due to the system structure (the topology of the system interaction graph).

Assessing whether all the systems with a given structure share a relevant structural property [4], [7], [16] helps explain how biological networks in nature preserve fundamental behaviours in the most diverse environmental conditions by design, independent of parameter values [4], [23], [27], [30], and also enables the rational design of robust artificial biomolecular networks in synthetic biology [9].

This paper focuses on the investigation of structurally signed input-output influences among the dynamic components of a biological network [17], [18], [19], [20], [21]. When a persistent positive input is applied to the system, the steadystate variation of the output variable can be either positive, negative, or zero: the influence is structural if the sign of the variation is independent of parameter values, while it is sign-indeterminate otherwise. The structural influence matrix can be built, whose $(i, j)$ entry is the structural sign of the steady-state variation of the $i$ th variable, when an additive

The work of Giulia Giordano was supported by the Aspasia Grant, $3 \mathrm{mE}$ Faculty, TU Delft.

${ }^{a}$ Dipartimento di Scienze Matematiche, Informatiche e Fisiche, Università degli Studi di Udine, 33100 Udine, Italy. blanchini@uniud. it

${ }^{b}$ Delft Center for Systems and Control, Delft University of Technology, 2628 CD Delft, The Netherlands. g.giordanodtudelft.nl input is applied to the $j$ th system equation. The influence matrix is traditionally employed in the ecological literature to understand communities of interacting species: a persistent perturbation is applied to a species in the community, to assess how the density of the various species changes at the new equilibrium [11], [12] (see also [18] and the references therein). This type of study was recently applied also to biomolecular systems, leading to novel approaches to assess signed steadystate sensitivities [29]. Research efforts are currently devoted to efficiently compute input-output influences [8], [25], [26].

Structural influence analysis enables us to predict steadystate behaviours that arise, regardless of the specific parameter values, in view of the system structure (when it is correctly known); while, given an uncertain 'conjectured' structure, the disagreement between structural predictions and experimental data allows for model falsification [17], [2].

To this aim, an algorithm was proposed in [21] to structurally compute steady-state input-output influences, and the steady-state influence matrix, for systems that are affine in both input and output, under the assumption of small input perturbations. The approach resorts to a linearised analysis and exploits the system local BDC-decomposition [5], [6].

In this paper, conversely, we consider a wide class of systems admitting a global $B D C$-decomposition and we assess the structural steady-state input-output influence globally, without assuming small signals and a linearised system. Our decomposition is suitable to describe generic systems, not necessarily affine in the input and the output. The main contributions are the following.

- A new algorithm is proposed to structurally compute global steady-state input-output influences, whose complexity is $2^{m-n}$ for a system with $m$ unknown parameters and $n$ state variables; in particular for large-scale systems, frequently encountered in the biological literature, this is a considerable improvement with respect to the complexity $2^{m}$ of the algorithm proposed in [21].

- When an influence is structurally zero, a test is proposed to discriminate between perfect adaptation (the output eventually goes back to the pre-perturbation value, after a non-trivial transient [1], [9], [30]) and the case in which the output is not influenced by the input, because the overall transfer function is zero.

- Also, when the uncertain parameters are subject to known interval bounds, a quantitative result provides the maximum and minimum output variations for a given input, enabling sensitivity analysis.

As shown in the concluding examples, our methods are able to reveal the structurally signed steady-state behaviours of biomolecular networks studied in the literature, and to identify proper perfect adaptation phenomena. 


\section{Problem Setup}

Consider a nonlinear dynamical system of the form

$$
\begin{aligned}
\dot{x}(t) & =S f(x(t), u(t)) \\
y(t) & =\operatorname{Rh}(x(t))
\end{aligned}
$$

with state $x \in \mathbb{R}^{n}$, input $u \in \mathbb{R}^{q}$ and output $y \in \mathbb{R}^{p}$, where the "stoichiometric" matrix $S \in \mathbb{Z}^{n \times r}$ and the output matrix $R \in \mathbb{R}^{p \times l}$ represent the known network structure, while $f$ and $h$ are unknown vector functions of size $r$ and $l$ respectively.

Assumption 1: There exist two convex sets $\mathcal{X} \subseteq \mathbb{R}^{n}$ and $\mathcal{U} \subseteq \mathbb{R}^{q}$, with non-empty interior $\operatorname{int}\{\mathcal{X}\}$ and $\operatorname{int}\{\mathcal{U}\}$, such that $f: \mathcal{X} \times \mathcal{U} \rightarrow \mathbb{R}^{r}$ and for all constant inputs $\bar{u} \in \operatorname{int}\{\mathcal{U}\}$ there exists a unique steady state $\bar{x} \in \operatorname{int}\{\mathcal{X}\}$, which is asymptotically stable.

For biological systems typically $\mathcal{X}=\mathbb{R}_{\geq 0}^{n}$ and $\mathcal{U}=\mathbb{R}_{>0}^{m}$, and we are interested in the effect of perturbations around a stable positive steady-state. We assume that, upon an input perturbation from $\bar{u}$ to $\bar{u}+\bar{v}$, the steady-state condition varies from $\bar{x}$ to $\bar{x}+\bar{z}$, leading to a steady-state output variation from $\bar{y}$ to $\bar{y}+\bar{w}$ :

$$
\left\{\begin{array} { l } 
{ 0 = S f ( \overline { x } , \overline { u } ) } \\
{ \overline { y } = \operatorname { R h } ( \overline { x } ) }
\end{array} \Rightarrow \left\{\begin{array}{l}
0=S f(\bar{x}+\bar{z}, \bar{u}+\bar{v}) \\
\bar{y}+\bar{w}=\operatorname{Rh}(\bar{x}+\bar{z})
\end{array}\right.\right.
$$

If $\bar{v}_{j} \neq 0$ and $\bar{v}_{k}=0$ for $k \neq j$, the $(i, j)$ steady-state inputoutput influence is defined as

$$
\Sigma_{i j}=\frac{\operatorname{sign}\left[\bar{w}_{i}\right]}{\operatorname{sign}\left[\bar{v}_{j}\right]}
$$

and the $(i, j)$ steady-state input-output sensitivity as

$$
\Theta_{i j}=\frac{\bar{w}_{i}}{\bar{v}_{j}}
$$

for $i \in\{1, \ldots, p\}$ and $j \in\{1, \ldots, q\}$. All these quantities, for a given model, depend on $\bar{v}$. If the model is known, standard software can determine the values of these quantities. However, we wish to structurally determine $\Sigma_{i j}$ or give bounds for $\Theta_{i j}$, when matrices $S$ and $R$ (describing the system structure) are given, while our knowledge of $f$ and $h$ is purely qualitative, based on the following assumptions.

Assumption 2: Monotonicity. All the component functions of $f$ and $h$ are continuously differentiable and all their partial derivatives are sign-definite (either positive or negative).

Assumption 3: Nonsingularity. The Jacobian $\partial f / \partial x$ is nonsingular, and in particular $\operatorname{det}[-\partial f / \partial x]>0$, for all $(x, u) \in \operatorname{int}\{\mathcal{X}\} \times \operatorname{int}\{\mathcal{U}\}$.

Stability is not our main concern here and is assumed; note that the positivity of $\operatorname{det}[-\partial f / \partial x]$ computed at the equilibrium is a necessary condition for local stability.

We begin by introducing some preliminary results (more details are in the Supplementary Information file). The first generalises the formula in [22, Exercise 3.23] (see also [16]).

Lemma 1: Given any continuously differentiable function $\varphi(\xi)$, defined on a convex set $\Xi$ with non-empty interior, and two points $\xi_{1}, \xi_{2} \in \Xi$, we have

$$
\varphi\left(\xi_{2}\right)-\varphi\left(\xi_{1}\right)=\left[\int_{0}^{1} \frac{\partial \varphi}{\partial \xi}\left(\xi_{1}+\sigma\left(\xi_{2}-\xi_{1}\right)\right) d \sigma\right]\left(\xi_{2}-\xi_{1}\right) .
$$

The second result extends the $B D C$-decomposition introduced in [5], [6], [21].

Lemma 2: The Jacobians of $S f$ and $R h$ evaluated at any point $(x, u) \in \mathcal{X} \times \mathcal{U}$ can be written as

$$
J_{f}=\left[B D_{x}(x, u) C \quad E D_{u}(x, u) F\right], \quad J_{h}=H D_{y}(x) L,
$$

where $B \in \mathbb{Z}^{n \times m_{x}}, C \in \mathbb{Z}^{m_{x} \times n}, E \in \mathbb{Z}^{n \times m_{u}}, F \in$ $\mathbb{Z}^{m_{u} \times q}, H \in \mathbb{Z}^{p \times m_{y}}, L \in \mathbb{Z}^{m_{y} \times n}$ are constant matrices, while $D_{x}(x, u) \in \mathbb{R}^{m_{x} \times m_{x}}, D_{u}(x, u) \in \mathbb{R}^{m_{u} \times m_{u}}$ and $D_{y}(x) \in \mathbb{R}^{m_{y} \times m_{y}}$ are positive-definite diagonal matrices, whose diagonal carries the absolute values of all the partial derivatives of $f$ with respect to $x\left(D_{x}\right)$, of $f$ with respect to $u\left(D_{u}\right)$ and of $h$ with respect to $x\left(D_{y}\right)$.

Matrices $B$ and $E$ are formed by (possibly repeated) columns of $S$, while $H$ is formed by (possibly repeated) columns of $R$. Matrices $C, F$ and $L$ have in their $i$ th row a \pm 1 in the position corresponding to the argument with respect to which the $i$ th derivative is taken $(+1$ if the derivative is strictly positive, -1 if it is strictly negative), and 0 elsewhere.

Example 1: Consider the biochemical reaction network

$$
\begin{aligned}
& A+B \stackrel{g_{a b}}{\underset{\hat{g}_{c}}{\longrightarrow}} C, A+C \stackrel{g_{a c}}{\longrightarrow} \emptyset, A \stackrel{g_{a}}{\longrightarrow} \emptyset, B \stackrel{g_{b}}{\longrightarrow} \emptyset, C \stackrel{g_{c}}{\longrightarrow} \emptyset, \\
& \emptyset \stackrel{u_{a}}{\longrightarrow} A, \emptyset \stackrel{u_{b}}{\longrightarrow} B, \emptyset \stackrel{u_{c}}{\longrightarrow} C
\end{aligned}
$$

Species concentrations are denoted with the corresponding lowercase letters and evolve according to equations (1)-(2), where $x=\left[\begin{array}{lll}a & b & c\end{array}\right]^{\top}$,

$$
\begin{aligned}
& S=\left[\begin{array}{ccccccccc}
-1 & 0 & 0 & -1 & -1 & 1 & 1 & 0 & 0 \\
0 & -1 & 0 & 0 & -1 & 1 & 0 & 1 & 0 \\
0 & 0 & -1 & -1 & 1 & -1 & 0 & 0 & 1
\end{array}\right] \\
& f(x, u)=\left[g_{a}(a) g_{b}(b) g_{c}(c) g_{a c}(a, c) g_{a b}(a, b) \hat{g}_{c}(c) u_{a} u_{b} u_{c}\right]^{\top} \text {, }
\end{aligned}
$$

where the $g$ functions (reaction rates) have strictly positive partial derivatives in each argument, $R$ is the identity matrix and $h(x)=x$. The system Jacobian with respect to $x$ is $J(x)=B D_{x}(x) C$, with

$$
\begin{aligned}
B & =\left[\begin{array}{cccccccc}
-1 & 0 & 0 & -1 & -1 & -1 & -1 & 1 \\
0 & -1 & 0 & 0 & 0 & -1 & -1 & 1 \\
0 & 0 & -1 & -1 & -1 & 1 & 1 & -1
\end{array}\right], \\
C & =\left[\begin{array}{cccccccc}
1 & 0 & 0 & 1 & 0 & 1 & 0 & 0 \\
0 & 1 & 0 & 0 & 0 & 0 & 1 & 0 \\
0 & 0 & 1 & 0 & 1 & 0 & 0 & 1
\end{array}\right]^{\top}, \\
D_{x} & =\operatorname{diag}\{\alpha, \beta, \gamma, \delta, \epsilon, \zeta, \eta, \theta\} \succ 0,
\end{aligned}
$$

where $\alpha=\partial g_{a} / \partial a, \beta=\partial g_{b} / \partial b, \gamma=\partial g_{c} / \partial c, \delta=\partial g_{a c} / \partial a$, $\epsilon=\partial g_{a c} / \partial c, \zeta=\partial g_{a b} / \partial a, \eta=\partial g_{a b} / \partial b, \theta=\partial \hat{g}_{c} / \partial c$. The system Jacobian with respect to $u$ and the Jacobian of $R h$ are constant, hence $D_{u}$ and $D_{y}$ are identity matrices. $\diamond$

Define the shifted variables $z(t)=x(t)-\bar{x}, v(t)=u(t)-\bar{u}$ and $w(t)=y(t)-\bar{y}$. From Lemma 2 we have the local BDCdecomposition: the linearised system at $(\bar{x}, \bar{u})$ is

$$
\begin{aligned}
\dot{z}(t) & =B D_{x} C z(t)+E D_{u} F v(t) \\
w(t) & =H D_{y} L z(t)
\end{aligned}
$$

where the diagonal matrices $D_{x}=D_{x}(\bar{x}, \bar{u}), D_{u}=D_{u}(\bar{x}, \bar{u})$ and $D_{y}=D_{y}(\bar{x})$ are constant. In view of Lemma 1 , we also have the global BDC-decomposition:

$$
\begin{aligned}
\dot{z}(t) & =B \Delta_{x}(z, v) C z(t)+E \Delta_{u}(z, v) F v(t) \\
w(t) & =H \Delta_{y}(z) L z(t)
\end{aligned}
$$


where $\Delta_{x}, \Delta_{u}$ and $\Delta_{y}$ are diagonal matrices with entries

$$
\Delta_{k}(z, v)=\int_{0}^{1} D_{k}(\bar{x}+\sigma z, \bar{u}+\sigma v) d \sigma .
$$

System (6)-(7) is an equivalent representation of (1)-(2).

If bounds $d_{k}^{-} \leq D_{k} \leq d_{k}^{+}$are available, they hold as well for $\Delta_{k}(z, v)$, which is the average of $D_{k}(x, u)$ on the segment originating in $(\bar{x}, \bar{u})$ and ending in $(\bar{x}+z, \bar{u}+v)$.

\section{Structural Influence AnAlysis}

Definition 1: Given a constant input perturbation $\bar{v}_{j} \neq 0$ $\left(\bar{v}_{k}=0\right.$ for $k \neq j$ ), leading to a steady-state variation $\bar{w}_{i}$ of the $i$ th output, the $(i, j)$ steady-state input-output influence $\Sigma_{i j}=\operatorname{sign}\left[\bar{w}_{i} / \bar{v}_{j}\right]$ is structurally positive ('+'), negative ('-') or zero (' 0 ') if it is positive, negative or zero for all possible choices of the diagonal entries $\Delta_{k}$ of the diagonal positive definite matrices $\Delta_{x}, \Delta_{u}$ and $\Delta_{y}$, while it is indeterminate ('?') if its sign depends on the $\Delta_{k}$. Hence, structurally,

$$
\Sigma_{i j} \in\{+,-, 0, ?\} .
$$

We assume, without restriction, that $u$ and $y$ are scalars, so that the input perturbation and the output steady-state variation are $\bar{v}, \bar{w} \in \mathbb{R}$. If $q>1$ and/or $p>1$, all the $q \cdot p$ input-output pairs can be considered separately to compose the structural steady-state influence matrix $\Sigma=\left[\Sigma_{i j}\right]$. Define the function

$$
\phi(\Delta) \doteq \operatorname{det}\left[\begin{array}{cc}
-B \Delta_{x} C & -E \Delta_{u} F \\
H \Delta_{y} L & 0
\end{array}\right]=\operatorname{det}[\hat{B} \Delta \hat{C}]
$$

where $\Delta \doteq \operatorname{diag}\left\{\Delta_{x}, \Delta_{u}, \Delta_{y}\right\}$ and

$$
\hat{B} \doteq\left[\begin{array}{ccc}
B & E & 0 \\
0 & 0 & H
\end{array}\right], \quad \hat{C} \doteq\left[\begin{array}{cc}
-C & 0 \\
0 & -F \\
L & 0
\end{array}\right]
$$

and the function

$$
\psi(\Delta) \doteq \operatorname{det}\left[-B \Delta_{x} C\right]
$$

Theorem 1: Given system (1)-(2) under Assumptions 2 and 3 , consider the input $\bar{u} \in \operatorname{int}\{\mathcal{U}\}$ along with the corresponding equilibrium state $\bar{x} \in \operatorname{int}\{\mathcal{X}\}$ and output $\bar{y}$. Then, the equilibrium value $\bar{x}$ is unique, as required in Assumption 1. Moreover, let $\bar{u}+\bar{v} \in \operatorname{int}\{\mathcal{U}\}$ be an input perturbation corresponding to the new equilibrium state $\bar{x}+\bar{z} \in \operatorname{int}\{\mathcal{X}\}$ (again unique) and output $\bar{y}+\bar{w}$. Then, the steady-state inputoutput influence is structurally positive (negative, zero) if and only if

$$
\phi(\Delta)>(<,=) \quad 0
$$

for all $\Delta \succ 0$. It is sign-indeterminate otherwise.

Proof: Uniqueness of the steady state $\bar{x}$ (as any other steady state $\bar{x}+\bar{z}$ ) can be proved with the argument in [6]: if $v=0$, equation (6) at steady-state becomes $0=B \Delta_{x} C z$, hence $z=0$ (namely, $x=\bar{x}$ ) is a steady state. If $x=\bar{x}+\tilde{z}$ were another steady-state, we would have $0=B \Delta_{x} C \tilde{z}$, which is impossible since $\psi(\Delta)=\operatorname{det}\left[-B \Delta_{x} C\right] \neq 0$ in view of Assumption 3. To prove the second part of the theorem, the steady-state conditions for (6)-(7) can be written as

$$
\left[\begin{array}{cc}
-B \Delta_{x} C & 0 \\
H \Delta_{y} L & 1
\end{array}\right]\left[\begin{array}{c}
\bar{z} \\
(-\bar{w})
\end{array}\right]=\left[\begin{array}{c}
-E \Delta_{u} F(-\bar{v}) \\
0
\end{array}\right]
$$

for some unknown positive diagonal matrices $\Delta_{x}, \Delta_{u}, \Delta_{y}$. If we apply Cramer's rule to derive $-\bar{w}$ as a function of $-\bar{v}$, adjusting the sign we get

$$
\bar{w}=\frac{\operatorname{det}\left[\begin{array}{cc}
-B \Delta_{x} C & -E \Delta_{u} F \\
H \Delta_{y} L & 0
\end{array}\right]}{\operatorname{det}\left[\begin{array}{cc}
-B \Delta_{x} C & 0 \\
H \Delta_{y} L & 1
\end{array}\right]} \bar{v}=\frac{\phi(\Delta)}{\psi(\Delta)} \bar{v} .
$$

Since $\psi(\Delta)>0$ (Assumption 3), the proof is concluded.

\section{Checking The Structural Sign of $\phi$ Requires COMPUTING $2^{m-n}$ DETERMINANTS}

Here, we consider the problem of determining the structural sign of $\phi(\Delta)=\operatorname{det}[\hat{B} \Delta \hat{C}]$, or of $\psi(\Delta)=\operatorname{det}\left[-B \Delta_{x} C\right]$ (which boils down to the same problem).

According to [21], we can check the sign of function $\phi(\Delta)$ for all diagonal matrices $\Delta=\operatorname{diag}\left\{\Delta_{1}, \Delta_{2}, \ldots, \Delta_{m}\right\} \succ 0$ by computing the function only at a finite number of points. Consider the set of binary strings of length $m$,

$$
\mathcal{K}_{m}=\left\{k=\left(k_{1}, k_{2}, \ldots k_{m}\right), k_{i} \in\{0,1\}\right\},
$$

associated with the vertices of the unit hyper-cube $0 \leq \Delta_{i} \leq 1, i=1,2 \ldots, m$.

Then, we have the following vertex algorithm [21]:

1) $\phi(\Delta)>0$ for all $\Delta \succ 0$ iff $\phi\left(k_{1}, k_{2}, \ldots, k_{m}\right) \geq 0$ for all $k \in \mathcal{K}_{m}$ and $\phi(1,1, \ldots, 1)>0$.

2) $\phi(\Delta)<0$ for all $\Delta \succ 0$ iff $\phi\left(k_{1}, k_{2}, \ldots, k_{m}\right) \leq 0$ for all $k \in \mathcal{K}_{m}$ and $\phi(1,1, \ldots, 1)<0$.

3) $\phi(\Delta)=0$ for all $\Delta \succ 0$ iff $\phi\left(k_{1}, k_{2}, \ldots, k_{m}\right)=0$ for all $k \in \mathcal{K}_{m}$.

4) $\phi(\Delta)$ is not sign determined iff it has different signs for two choices of $k \in \mathcal{K}_{m}$.

Example 2: For the biochemical reaction network in Example 1, the vertex algorithm allows us to check that $\operatorname{det}\left[-B \Delta_{x} C\right]>0$ structurally. To assess the steady-state influence $\Sigma_{i j}$ of the input $j \in\{1,2,3\}$ on the output $i \in\{1,2,3\}$ we need to check the sign of

$$
\phi(\Delta)=\operatorname{det}\left[\begin{array}{ccc|c}
\zeta+\delta+\alpha & \eta & -\theta+\epsilon & -E_{1} \\
\zeta & \eta+\beta & -\theta & -E_{2} \\
-\zeta+\delta & -\eta & \theta+\epsilon+\gamma & -E_{3} \\
\hline H_{1} & H_{2} & H_{3} & 0
\end{array}\right],
$$

where only $E_{j}$ and $H_{i}$ are equal to 1 , while the other entries of the input column vector and of the output row vector are zero. By considering all the 9 input-output pairs we can compose the structural steady-state influence matrix

$$
\Sigma=\left[\Sigma_{i j}\right]=\left[\begin{array}{lll}
+ & - & ? \\
- & + & + \\
? & + & +
\end{array}\right]
$$

In general, to assess the structural sign of an input-output influence, the algorithm requires computing $2^{m}$ determinants. We show here that, for a significant class of networks, this complexity can be reduced to $2^{m-n}$. To explain the idea that we will formalise next, consider the problem of checking the sign of $\operatorname{det}\left[-B \Delta_{x} C\right]$ for the network in Example 1. To reduce complexity, we can divide all columns by one of the free parameters and check the sign of 


$$
\psi^{\prime}\left(\delta^{\prime}, \epsilon^{\prime}, \zeta^{\prime}, \eta^{\prime}, \theta^{\prime}\right)=\operatorname{det}\left[\begin{array}{ccc}
\zeta^{\prime}+\delta^{\prime}+1 & -\eta^{\prime} & -\theta^{\prime}+\epsilon^{\prime} \\
\zeta^{\prime} & \eta^{\prime}+1 & -\theta^{\prime} \\
-\zeta^{\prime}+\delta^{\prime} & -\eta^{\prime} & \theta^{\prime}+\epsilon^{\prime}+1
\end{array}\right],
$$

where the parameters are now $5\left(\delta^{\prime}=\delta / \alpha, \epsilon^{\prime}=\epsilon / \gamma, \zeta^{\prime}=\right.$ $\left.\zeta / \alpha, \eta^{\prime}=\eta / \beta, \theta^{\prime}=\theta / \gamma\right)$ instead of 8 . For large-scale systems, the reduction in computational complexity is noteworthy.

However, a technical problems arises: it is impossible to apply the vertex algorithm proposed in [21] to the polynomial $\psi^{\prime}$. To explain this key point, let us introduce some definitions.

Definition 2: A function $\varphi(\Delta)$ is multi-affine if it is affine in each argument.

Definition 3: A function $\varphi(\Delta)$ is homogeneous of degree $d$ if $\varphi(k \Delta)=k^{d} \varphi(\Delta)$. In particular, a polynomial is homogeneous if all its nonzero terms have the same degree $d$. $\diamond$ Function $\varphi(\alpha, \beta, \gamma)=1+\alpha+\beta-\alpha \beta+\alpha \beta \gamma$ is multi-affine, but not homogeneous. The following proposition from [3] holds.

Proposition 1: A multi-affine function $\varphi(\Delta)$ defined on a hyper-rectangle $\Delta_{i}^{-} \leq \Delta_{i} \leq \Delta_{i}^{+}$reaches its minimum and its maximum on the vertices of the hyper-rectangle.

The algorithm in [21] relies on the above proposition and on the possibility to restrict the analysis to the unit hypercube, since the original polynomials $\phi(\Delta)$ and $\psi(\Delta)$ are multi-affine and homogeneous. Yet, the new polynomials $\phi^{\prime}$ and $\psi^{\prime}$ obtained by dividing each column by one of the free parameters are multi-affine, but not homogeneous, hence the vertex algorithm described above cannot be applied. For example, $\varphi(\alpha, \beta, \gamma)=1+\alpha+\beta-\alpha \beta+\alpha \beta \gamma$ is positive on all the vertices of the hyper-cube $\alpha, \beta, \gamma \in\{0,1\}$, but it is not positive for all $\alpha, \beta, \gamma>0$. Therefore, we cannot look at the unit hyper-cube and we need to face a new problem.

Problem: Given the multi-affine, possibly nonhomogeneous, polynomial $\varphi(\Delta)$, check if $\varphi(\Delta)$ always has the same sign for all $\Delta \succ 0$ by computing $\varphi(\Delta)$ at a finite number of points.

Consider a multi-affine polynomial $\varphi(\Delta)$ depending on $m$ variables: $\Delta_{1}, \ldots, \Delta_{m}$. Given $k \in \mathcal{K}_{m}$, as in (11), and a real number $M>0$, we define $M k$ as the string whose elements are $M k_{i} \in\{0, M\}$. Given two strings $h, k \in \mathcal{K}_{m}, h \leq k$ means that $h_{i} \leq k_{i}$ for all $i=1, \ldots, m$ (e.g., $(1,0,0,1) \leq$ $(1,0,1,1))$. Then, we can write

$$
\varphi(\Delta)=\sum_{k \in \mathcal{K}_{m}} \varphi_{k} \Delta_{1}^{k_{1}} \Delta_{2}^{k_{2}} \ldots \Delta_{m}^{k_{m}}
$$

and define the polynomial of a single variable $M$

$$
\varphi(M k) \doteq \sum_{h \leq k} \varphi_{h} M^{h_{1}+h_{2}+\cdots+h_{m}} .
$$

The following theorem plays an important role.

Theorem 2: Given the multi-affine polynomial $\varphi(\Delta)$, with $\Delta=\operatorname{diag}\left\{\Delta_{1}, \ldots, \Delta_{m}\right\}$, these statements are equivalent:

i) $\varphi(\Delta) \geq 0$ for all $\Delta \succeq 0$.

ii) $\varphi_{k} \geq 0$ for all $k \in \mathcal{K}_{m}$.

iii) $\quad \varphi(M k) \geq 0$ for all $k \in \mathcal{K}_{m}$ and $M \geq 0$.

iv) The leading coefficient (associated with the highest power) of $\varphi(M k)$ is nonnegative for all $k \in \mathcal{K}_{m}$ and $M \geq 0$.
Remark 1: Statements iii) and iv) would not be equivalent for a single polynomial, but they are equivalent if we consider all possible choices of $k$.

Proof: Obviously i $\Rightarrow$ ii) $\Rightarrow$ iii) $\Rightarrow$ iv).

iii) $\Rightarrow$ i): function $\varphi(\Delta)$ is multi-affine, hence on the domain $0 \leq \Delta_{i} \leq M$ it reaches its extrema at the vertices, according to Proposition 1. So $\varphi(\Delta)$ is nonnegative in the whole domain iff its value at all the vertices $\varphi(M k)$ is nonnegative.

iv $\Rightarrow$ ii): it follows from the fact that each coefficient $\varphi_{k}$ in (13) becomes leading for a proper choice of $k$, by taking $k$ with ones only corresponding to the variables in the monomial associated with the coefficient $\varphi_{k}$, and zero elsewhere.

Then, based on the theorem, we can devise an algorithm with reduced complexity that solves the problem for a significant class of systems satisfying the following assumption.

Assumption 4: Each row of matrix $C$ has a single nonzero entry, equal either to +1 or to -1 .

$\diamond$

The new matrix $\Psi$ obtained from $B \Delta_{x} C$ after reducing the number of parameters by column-wise division has columns of the form

$$
\Psi_{\bullet j}=V_{j 0}+\hat{\Delta}_{1} V_{j 1}+\cdots+\hat{\Delta}_{m-n} V_{j, m-n},
$$

where $V_{j i}$ are constant column vectors with integer entries, while $\hat{\Delta}_{i}$ are the rescaled free parameters, which are $m-n$ in total. Let us denote by $\Psi^{(k)}$ the matrix obtained by choosing $\hat{\Delta}=\operatorname{diag}\{k\}$, for some $k \in \mathcal{K}_{m-n}$.

The leading coefficients are determined as follows.

\section{Procedure 1: Computation of the leading coefficients.}

- For all $k \in \mathcal{K}_{m-n}$, consider matrix $\Psi^{(k)}$, whose column $\Psi_{\bullet j}^{(k)}$ is formed as follows.

- If, corresponding to string $k$, all parameters $\hat{\Delta}_{i}$ appearing in the $j$ th column are zero, then $\Psi_{\bullet j}^{(k)}=V_{j 0}$.

- If, corresponding to string $k$, at least one parameter appearing in the $j$ th column is $\hat{\Delta}_{i}=M$, then $\Psi_{\bullet j}^{(k)}=$ $\frac{\hat{\Delta}_{1}}{M} V_{j 1}+\cdots+\frac{\hat{\Delta}_{m-n}}{M} V_{j, m-n}$ (i.e., $V_{j 0}$ is neglected).

- Then, the leading coefficient is computed as $\operatorname{det}\left[\Psi^{(k)}\right]$.

For instance, consider the polynomial in (12), with $\delta^{\prime}=M$, $\epsilon^{\prime}=0, \zeta^{\prime}=M, \eta^{\prime}=0, \theta^{\prime}=M$, hence $k=(1,0,1,0,1)$. The leading coefficient, associated with the term $M^{2}$, is

$$
\operatorname{det}\left[\Psi^{(k)}\right]=\operatorname{det}\left[\begin{array}{ccc}
1+1+0 & -0 & -1+0 \\
1 & 0+1 & -1 \\
-1+1 & -0 & 1+0+0
\end{array}\right] .
$$

Based on Theorem 2 and on the above observations, the next result can be proved.

Proposition 2: For the multi-affine polynomial $\varphi(\Delta)$, with $\Delta=\operatorname{diag}\left\{\Delta_{1}, \ldots, \Delta_{m-n}\right\}$, the following statements hold.

i) $\varphi(\Delta) \geq 0$ (resp. $\varphi(\Delta) \leq 0$ ) iff all the leading coefficients computed with Procedure 1 are nonnegative (nonpositive). Strict inequality $(>$ or $<$ ) requires that at least one leading coefficient is strictly positive or negative.

ii) $\varphi(\Delta) \equiv 0$ iff all the leading coefficients are zero.

iii) The number of leading coefficients is $2^{m-n}$.

Remark 2: The complexity depends on $m-n$, the difference between the number of free parameters and of state variables, which is expected to be relatively small even for 
large networks. This strongly reduces the computational effort. $\diamond$

\section{Perfect Adaptation Versus Disconnection}

Perfect adaptation [1], [9] occurs when a persistent input perturbation is applied to the system and, after a non-trivial transient, the output asymptotically gets back to its preperturbation value: hence, the steady-state influence is zero. This phenomenon is often observed in biological systems, including bacterial chemotaxis [30], calcium homeostasis [14], osmoregulation [24], and cell sensory systems [28]. Yet, the influence is zero also if the input-output transfer function is identically zero (disconnection), typically due to a lack of reachability or of observability. Perfect adaptation reveals the ability to maintain biological homeostasis, while disconnection is not considered adaptation. To structurally distinguish between the two different phenomena (cf. Section VII-A), note that $\phi(\Delta)$ is the numerator of the transfer function

$$
\phi(\Delta, s)=\operatorname{det}\left[\begin{array}{cc}
s I-B \Delta_{x} C & -E \Delta_{u} F \\
H \Delta_{y} L & 0
\end{array}\right]
$$

computed at $s=0$. If $\phi(\Delta)=0$, the influence is zero. We can structurally assess whether the whole polynomial is identically zero, in view of the following result.

Proposition 3: The polynomial $\phi(\Delta, s)$ in (15) is structurally zero iff it is zero, for all possible choices of $\Delta \succ 0$, for at least $n+1$ different choices of $s: s_{1}, s_{2}, \ldots, s_{n+1}$. $\square$ The vertex algorithm allows to check if $\phi\left(\Delta, s_{k}\right)=0$ for all $\Delta \succ 0$, and thus to discriminate between perfect adaptation and input-output disconnection (i.e., $\phi\left(\Delta, s_{k}\right)=0$ not only when $s_{k}=0$, but also for other $n$ choices of $s_{k}$ ).

\section{InPut-OUtPUt SEnSitivity AnAlysis}

We can adopt a more quantitative approach to give robust bounds on the quantity $\Theta=y / u$, without requiring it to be sign definite. Assume that $\Delta$ is bounded in a hyper-rectangle

$$
\Delta \in \mathcal{R}=\left\{\Delta: \Delta_{i}^{-} \leq \Delta_{i} \leq \Delta_{i}^{+}, i=1,2, \ldots m\right\},
$$

where $\Delta_{i}^{-}$and $\Delta_{i}^{+}$are known. We define the vertex set

$$
\mathcal{V}=\left\{\Delta: \Delta_{i} \in\left\{\Delta_{i}^{-}, \Delta_{i}^{+}\right\}, i=1,2, \ldots m\right\} .
$$

In view of (10), we need bounds for the ratio $\phi(\Delta) / \psi(\Delta)$.

Theorem 3: Assume $\psi(\Delta) \neq 0$ for all $\Delta \in \mathcal{R}$. Then, for all $\phi(\Delta) / \psi(\Delta)$ with $\Delta \in \mathcal{R}$, we have

$$
\min _{\Delta \in \mathcal{V}} \frac{\phi(\Delta)}{\psi(\Delta)} \leq \frac{\phi(\Delta)}{\psi(\Delta)} \leq \max _{\Delta \in \mathcal{V}} \frac{\phi(\Delta)}{\psi(\Delta)}
$$

The bounds are tight (the true minimum and maximum).

Proof: For the upper bound (the proof for the lower bound is identical), we have that $\phi(\Delta) / \psi(\Delta) \leq \mu$ iff $\rho(\Delta, \mu)=\phi(\Delta)-\mu \psi(\Delta) \leq 0$, for all $\Delta \in \mathcal{R}$. Since the functions $\phi(\Delta)$ and $\psi(\Delta)$ are multi-affine, the condition is equivalent to $\rho(\Delta, \mu) \leq 0$ for all $\Delta \in \mathcal{V}$, in turn equivalent to $\phi(\Delta) / \psi(\Delta) \leq \mu$ for all $\Delta \in \mathcal{V}$.

To apply the theorem, we need to compute the function value at $2^{m}$ points, corresponding to all the vertices.

Example 3: Consider again the network of Example 1. Assume that, for all parameters, $1 \leq \Delta_{i} \leq 10$. The matrix
$\Theta=\left[\Theta_{i j}\right]$ obtained by considering all input-output pairs has element-wise lower and upper bounds given by

$$
\left[\begin{array}{ccc}
{[0.034,0.78]} & {[-1.35,-0.0008]} & {[-0.70,0.25]} \\
{[-0.67,-0.0045]} & {[0.055,0.95]} & {[0.0045,0.67]} \\
{[-0.70,0.25]} & {[0.0008,1.35]} & {[0.034,0.78]}
\end{array}\right],
$$

fully consistent with the influence matrix $\Sigma$, since only the entries $(1,3)$ and $(3,1)$ have bounds with opposite signs. $\diamond$

\section{BIOLOGICAL EXAMPLES}

\section{A. An enzyme-driven biomolecular network}

In the biomolecular network in [10, pp. 208-209], the enzyme $A$ catalyses the conversion of substrates $B$ and $C$ into the product $P$, by means of the formation of the intermediate complexes $D, E$ and $F$ : the chemical reactions are

$$
\begin{array}{r}
A+B \underset{g_{d}}{\stackrel{g_{a b}}{\rightleftharpoons}} D, A+C \underset{g_{e}}{\stackrel{g_{a c}}{\rightleftharpoons}} E, C+D \underset{g_{f}}{\stackrel{g_{c d}}{\rightleftharpoons}} F \underset{g_{b e}}{\stackrel{\hat{g}_{f}}{\rightleftharpoons}} B+E, \\
F \stackrel{\tilde{g}_{f}}{\longrightarrow} A+P, \emptyset \stackrel{u_{b}}{\longrightarrow} B \stackrel{g_{b}}{\longrightarrow} \emptyset, \emptyset \stackrel{u_{c}}{\longrightarrow} C \stackrel{g_{c}}{\longrightarrow} \emptyset, P \stackrel{g_{p}}{\longrightarrow} \emptyset .
\end{array}
$$

If we also consider the additional reactions $\emptyset \stackrel{u_{a}}{\longrightarrow} A \stackrel{g_{a}}{\longrightarrow} \emptyset$, the associated system of differential equations is

$$
\begin{aligned}
\dot{a} & =-g_{a b}(a, b)+g_{d}(d)-g_{a c}(a, c)+g_{e}(e)+\tilde{g}_{f}(f)-g_{a}(a)+u_{a} \\
\dot{b} & =-g_{a b}(a, b)+g_{d}(d)-g_{b e}(b, e)+\hat{g}_{f}(f)-g_{b}(b)+u_{b} \\
\dot{c} & =-g_{a c}(a, c)+g_{e}(e)-g_{c d}(c, d)+g_{f}(f)-g_{c}(c)+u_{c} \\
\dot{d} & =g_{a b}(a, b)-g_{d}(d)-g_{c d}(c, d)+g_{f}(f) \\
\dot{e} & =g_{a c}(a, c)-g_{e}(e)-g_{b e}(b, e)+\hat{g}_{f}(f) \\
\dot{f} & =g_{b e}(b, e)+g_{c d}(c, d)-g_{f}(f)-\hat{g}_{f}(f)-\tilde{g}_{f}(f) \\
\dot{p} & =\tilde{g}_{f}(f)-g_{p}(p)
\end{aligned}
$$

The system has $n=7$ state variables and matrix $S \in \mathbb{Z}^{7 \times 16}$, since there are 13 reaction-rate functions, which have strictly positive derivative in each argument and are zero iff at least one argument is zero, and 3 external inputs, $u_{a}, u_{b}$ and $u_{c}$. The unique equilibrium is positive, hence, if it is stable, Assumption 1 is satisfied in the positive orthants. The Supplementary Information file reports the system $B D C$ decomposition, where $D_{x}$ has 17 positive diagonal entries. The algorithm reveals that $\operatorname{det}\left[-B \Delta_{x} C\right]>0$, for all $\Delta_{x} \succ 0$. The influence matrix is

$$
\Sigma=\left[\begin{array}{ccccccc}
+ & 0 & 0 & + & + & + & 0 \\
- & + & - & ? & - & ? & 0 \\
- & - & + & - & ? & ? & 0 \\
? & + & ? & ? & ? & ? & 0 \\
? & ? & + & ? & ? & ? & 0 \\
+ & + & + & + & + & + & 0 \\
+ & + & + & + & + & + & +
\end{array}\right]
$$

and 35 out of 49 entries are structurally signed. Of them, 8 are zero. To distinguish between perfect adaptation and disconnection (cf. Section V, Proposition 3), we compute the numerator of the corresponding transfer functions at 7 different points: the first six 0 entries of column $\Sigma_{\bullet}$ are due to disconnection (the variables $a$ to $f$ are not affected by an input applied to the equation of $p$, also during the transient), while the 0 entries $\Sigma_{12}$ and $\Sigma_{13}$ reveal actual perfect adaptation.

The novel algorithm significantly reduces the computational complexity: to structurally assess $\Sigma_{i j}$, it is enough to compute $2^{17-7}=2^{10}=1024$ determinants, instead of $2^{17}=131072$. The sensitivity analysis, reported in the Supplementary Information file, shows full consistency with the signed matrix $\Sigma$. 


\section{B. mRNA-protein dynamics in a multicellular network}

A multicellular network of repressilators (genetic oscillators, [13]) coupled by quorum sensing is discussed in [15]. Each repressilator involves three genes $\left(X_{1}, X_{2}\right.$ and $\left.X_{3}\right)$, each expressing a protein $\left(X_{4}, X_{5}\right.$ and $X_{6}$ respectively) that inhibits the transcription of another gene, in a negative feedback loop. Coupling through quorum sensing is enabled by cellto-cell communication based on the autoinducer $\left(X_{7}\right.$, a small molecule able to diffuse through the cell membrane), synthesised thanks to protein $X_{4}$, that activates the transcription of gene $X_{3}$. The system describing mRNA and protein evolution in each of the cells is [15], [10, pp. 314-315]:

$$
\begin{array}{ll}
\dot{x}_{1}=-\alpha x_{1}+g_{6}\left(x_{6}\right) & \dot{x}_{2}=-\beta x_{2}+g_{4}\left(x_{4}\right) \\
\dot{x}_{3}=-\gamma x_{3}+g_{5}\left(x_{5}\right)+g_{7}\left(x_{7}\right) & \dot{x}_{4}=-\delta x_{4}+\pi x_{1} \\
\dot{x}_{5}=-\varepsilon x_{5}+\rho x_{2} & \dot{x}_{6}=-\phi x_{6}+\sigma x_{3} \\
\dot{x}_{7}=\tau x_{4}-\omega x_{7}+\eta\left(u_{7}-x_{7}\right) &
\end{array}
$$

where $g_{4}, g_{5}, g_{6}$ are decreasing Hill-type functions, $g_{7}$ is an increasing Michaelis-Menten function, and $u_{7}$ denotes the average extracellular concentration of the autoinducer. The system has $n=7$ state variables and $S \in \mathbb{Z}^{7 \times 17}$, due to the presence of 16 reaction-rate functions and 1 external input, $u_{7}$. The unique equilibrium is positive, hence, if it is stable, Assumption 1 is satisfied in the positive orthants. The system admits a $B D C$-decomposition where $D_{x}$ has 16 positive diagonal entries, as reported in the Supplementary Information file. Also in this case, $\operatorname{det}\left[-B \Delta_{x} C\right]>0$ structurally, for all possible $\Delta_{x} \succ 0$. The steady-state influence matrix is

$$
\Sigma=\left[\begin{array}{ccccccc}
+ & + & - & - & + & - & - \\
- & + & + & - & - & + & + \\
+ & - & + & + & - & - & + \\
+ & + & - & + & + & - & - \\
- & + & + & - & + & + & + \\
+ & - & + & + & - & + & + \\
+ & + & - & + & + & - & +
\end{array}\right] .
$$

All the entries of the influence matrix are structurally signed (either positive or negative), regardless of the chosen parameters. The new algorithm requires to compute $2^{16-7}=2^{9}=$ 512 determinants, instead of $2^{16}=65536$. The Supplementary Information file reports the input-output sensitivities, fully consistent with the influence matrix $\Sigma$.

\section{CONCLUSIONS}

The global BDC-decomposition has enabled us to structurally assess signed steady-state input-output influences as global properties, through a novel algorithm with high computational efficiency. When an influence is structurally zero, we have given a criterion to discriminate between perfect adaptation and disconnection (identically zero transfer function). We have provided tight bounds for input-output sensitivities, when the uncertain parameters have known bounds. Our algorithm, applied to biomolecular systems from the literature, has revealed signed behaviours that exclusively rely on the system structure.

\section{REFERENCES}

[1] U. Alon, An Introduction to Systems Biology: Design Principles of Biological Circuits, Chapman \& Hall/CRC, 2006.
[2] D. Angeli and E. D. Sontag, "Remarks on the invalidation of biological models using monotone systems theory", 51st IEEE Conf. on Dec. and Control (CDC), 2012, pp. 2989-2994.

[3] B. R. Barmish, New Tools for Robustness of Linear Systems. Macmillan, 1994.

[4] F. Blanchini and E. Franco, "Structurally robust biological networks", BMC Systems Biology, 5(1):74, 2011.

[5] F. Blanchini and G. Giordano, "Piecewise-linear Lyapunov functions for structural stability of biochemical networks", Automatica, 50(10):24822493, 2014.

[6] F. Blanchini and G. Giordano, "Polyhedral Lyapunov functions structurally ensure global asymptotic stability of dynamical networks iff the Jacobian is non-singular", Automatica, 86(12):183-191, 2017.

[7] F. Blanchini, E. Franco, G. Giordano, "Determining the structural properties of a class of biological models", IEEE Conference on Decision and Control, pp. 5505-5510, 2012.

[8] B. Brehm and B. Fiedler, "Sensitivity of chemical reaction networks: A structural approach. 3. Regular multimolecular systems", Mathematical Methods in the Applied Sciences, 41(4):1344-1376, 2018.

[9] C. Briat, A. Gupta, M. Khammash, "Antithetic integral feedback ensures robust perfect adaptation in noisy biomolecular networks", Cell Systems, 2(1): 15-26, 2016.

[10] L. Chen, R. Wang, C. Li, and K. Aihara, Modeling Biomolecular Networks in Cells, Springer-Verlag, London, 2010.

[11] J. Dambacher, H. Li, and P. Rossignol, "Relevance of community structure in assessing indeterminacy of ecological predictions", Ecology, 83(5):1372-1385, 2002.

[12] J. M. Dambacher and R. Ramos Jiliberto, "Understanding and predicting effects of modified interactions through a qualitative analysis of community structure", The Quarterly Review of Biology, 82(3):227-250, 2007.

[13] M. Elowitz and S. Leibler, "A synthetic oscillatory network of transcriptional regulators", Nature, 403:335-338, 2000.

[14] H. El-Samad, J. P. Goff JP, M. Khammash, "Calcium homeostasis and parturient hypocalcemia: an integral feedback perspective", J. Theor. Biol., 214:17-29, 2002.

[15] J. Garcia-Ojalvo, M. Elowitz, and S. H. Strogatz, "Modeling a synthetic multicellular clock: Repressilators coupled by quorum sensing", Proc Natl Acad Sci U.S.A., 101:10955-10960, 2004.

[16] G. Giordano, Structural Analysis and Control of Dynamical Networks, Ph.D. thesis, University of Udine, Italy, 2016.

[17] G. Giordano, "CERT-mediated ceramide transfer is a structurally tunable flow-inducing mechanism with structural feed-forward loops", Royal Society Open Science, 5(6):180494, 2018.

[18] G. Giordano, C. Altafini, "Qualitative and quantitative responses to press perturbations in ecological networks", Scientific Reports, 7:11378, 2017.

[19] G. Giordano, C. Altafini, "Interaction sign patterns in biological networks: from qualitative to quantitative criteria", IEEE Conference on Decision and Control, pp. 5348-5353, 2017.

[20] G. Giordano, E. Franco, "Negative feedback enables structurally signed steady-state influences in artificial biomolecular networks", IEEE Conference on Decision and Control, pp. 3369-3374, 2016.

[21] G. Giordano, C. Cuba Samaniego, E. Franco, F. Blanchini, "Computing the structural influence matrix for biological systems", Journal of Mathematical Biology, 72(7):1927-1958, 2016.

[22] H. K. Khalil, Nonlinear systems. Prentice Hall, 2002.

[23] H. Kitano, "Biological robustness", Nature Reviews Genetics, 5(11):826-837, 2004.

[24] D. Muzzey, C. A. Gomez-Uribe, J. T. Mettetal, A. Van Oudenaarden, "Systems-level analysis of perfect adaptation in yeast osmoregulation", Cell, 138:160-171, 2009.

[25] D. Koslicki and M. Novak, "Exact probabilities for the indeterminacy of complex networks as perceived through press perturbations", Journal of Mathematical Biology, 76(4):877-909, 2018.

[26] J. Lages, D. L. Shepelyansky, and A. Zinovyev, "Inferring hidden causal relations between pathway members using reduced Google matrix of directed biological networks", PLOS ONE, 13(1):e0190812, 2018.

[27] G. Shinar and M. Feinberg, "Structural sources of robustness in biochemical reaction networks", Science, 327(5971):1389-1391, 2010.

[28] O. Shoval, L. Goentoro, Y. Hart, A. Mayo, E. D. Sontag, U. Alon, "Foldchange detection and scalar symmetry of sensory input fields", Proc Natl Acad Sci U.S.A., 107:15995-16000, 2010.

[29] E. D. Sontag, "A technique for determining the signs of sensitivities of steady states in chemical reaction networks", IET Systems Biology, 8(6):251-267, 2014

[30] T. M. Yi, Y. Huang, M. I. Simon, and J. Doyle, "Robust perfect adaptation in bacterial chemotaxis through integral feedback control", Proc Natl Acad Sci U.S.A., 97(9):4649-4653, 2000 


\title{
BDC-decomposition for global influence analysis - Supplementary Material
}

\author{
Franco Blanchini and Giulia Giordano
}

\section{Summary:}

In this Supplementary Information file, we provide additional details on the $B D C$-decomposition and we report some examples of influence analysis for real biomolecular systems, to showcase the proposed approach. All the necessary theory is reported, proved and thoroughly discussed in the main paper [3]. The examples broadly discussed here are complementary to the material in the example section of [3].

\section{Local and global $B D C$-decomposition: an overview}

In this section, we summarise fundamental information on the local and global $B D C$-decomposition $[1,2,6,11]$, including a detailed explanation of the computation of the $B D C$ matrices.

Consider a nonlinear system of the form

$$
\dot{x}(t)=S f(x(t))+f_{0},
$$

where $x \in \mathcal{D} \subseteq \mathbb{R}^{n}, S \in \mathbb{Z}^{n \times r}$ can be regarded as the stoichiometric or flow matrix of the system, $f: \mathcal{D} \subseteq \mathbb{R}^{n} \rightarrow \mathbb{R}^{r}$ is a vector of continuously differentiable functions with sign-definite partial derivatives, $\mathcal{D}$ is an open, convex domain, and $f_{0} \in \mathbb{R}^{n}$ is a constant vector. The equilibrium point $\bar{x}$ is such that $S f(\bar{x})+f_{0}=0$.

This class of models includes any chemical reaction network, or any phenomenological biomolecular model (e.g., gene regulatory models, signalling networks, etc.) that can be written as an equivalent chemical reaction network; also models typically used in ecology and population dynamics can be rewritten as in (1). In these cases, $\mathcal{D}=\mathbb{R}_{\geq 0}^{n}$.

Definition 1 System (1) admits a BDC-decomposition iff, for any $x \in \mathcal{D}$, the system Jacobian $J(x)=\partial(S f) / \partial x$ can be written as the positive linear combination of rank-one matrices, namely

$$
J(x)=\sum_{h=1}^{q} B_{h} D_{h}(x) C_{h}^{\top}=\sum_{h=1}^{q} R_{h} D_{h}(x),
$$

where $B_{h}$ and $C_{h}^{\top}$ are column and row vectors, respectively, so that $R_{h}=\left[B_{h} C_{h}^{\top}\right]$ are constant rank-one matrices (a rank-one matrix $R_{h}$ can always be written as the product of a column vector $B_{h}$ and a row vector $\left.C_{h}^{\top}\right)$, while $D_{h}(x)$, $h=1, \ldots, q$, are positive scalar functions depending on $x$.

In a compact form, $J(x)=B D(x) C$, where $D(x)$ is a diagonal matrix with positive diagonal entries $D_{h}(x), B$ is the matrix formed by the columns $B_{h}$ and $C$ is the matrix formed by the rows $C_{h}^{\top}$.

The above definition holds for any $x$ in the domain $\mathcal{D}$, hence, in particular, for any equilibrium point $\bar{x} \in \mathcal{D}$.

Systems of the form (1) always admit a $B D C$-decomposition, as shown in [6,11]. Matrices $B$ and $C$ can be built systematically, based on matrix $S$ and on qualitative information about $f(\cdot)$.

Proposition 1 Any system in the class (1) admits a BDC-decomposition, $J(x)=B D(x) C$, according to Definition 1.

Proof sketch The statement is proved constructively in $[6,11]$ : equation (1) is rewritten as $\dot{x}=\sum_{j=1}^{s} S_{j} f_{j}(x)+f_{0}$,

where $S_{j}$ is the $j$ th column of matrix $S$. The corresponding Jacobian is $J(x)=\sum_{j=1}^{s} S_{j}\left[\begin{array}{llll}\frac{\partial f_{j}}{\partial x_{1}} & \frac{\partial f_{j}}{\partial x_{2}} & \cdots & \frac{\partial f_{j}}{\partial x_{n}}\end{array}\right]$. Denoting by $D_{1}(x), D_{2}(x), \ldots, D_{q}(x)$ the absolute values of all the non-zero partial derivatives, it can be written $J(x)=\sum_{h=1}^{q} B_{h} D_{h}(x) C_{h}^{\top}$, where

- $D_{h}(x)=\left|\frac{\partial f_{j}}{\partial x_{i}}\right|$ for some $i$ and $j$;

- $B_{h}=S_{j}$, the column of $S$ associated with $f_{j}$;

- $C_{h}^{\top}$ has a single non-zero entry in the $i$ th position, equal to the sign of $\frac{\partial f_{j}}{\partial x_{i}}$. 


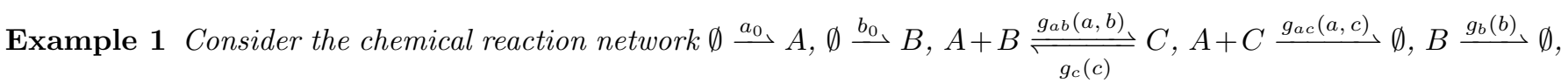
corresponding to the system

$$
\left\{\begin{array}{l}
\dot{a}=a_{0}-g_{a b}(a, b)-g_{a c}(a, c)+g_{c}(c) \\
\dot{b}=b_{0}-g_{a b}(a, b)-g_{b}(b)+g_{c}(c) \\
\dot{c}=g_{a b}(a, b)-g_{a c}(a, c)-g_{c}(c)
\end{array}\right.
$$

which can be rewritten as in model (1) by setting $x=\left[\begin{array}{lll}a & b & c\end{array}\right]^{\top}$,

$$
S=\left[\begin{array}{rrrr}
-1 & 1 & -1 & 0 \\
-1 & 1 & 0 & -1 \\
1 & -1 & -1 & 0
\end{array}\right], f(x)=\left[\begin{array}{c}
g_{a b}(a, b) \\
g_{c}(c) \\
g_{a c}(a, c) \\
g_{b}(b)
\end{array}\right], f_{0}=\left[\begin{array}{c}
a_{0} \\
b_{0} \\
0
\end{array}\right]
$$

Denoting the positive partial derivatives as $\alpha=\frac{\partial g_{a b}(a, b)}{\partial a}, \beta=\frac{\partial g_{a b}(a, b)}{\partial b}, \gamma=\frac{\partial g_{c}(c)}{\partial c}, \delta=\frac{\partial g_{a c}(a, c)}{\partial a}, \epsilon=\frac{\partial g_{a c}(a, c)}{\partial c}$, $\zeta=\frac{\partial g_{b}(b)}{\partial b}$, the Jacobian matrix and its BDC-decomposition are

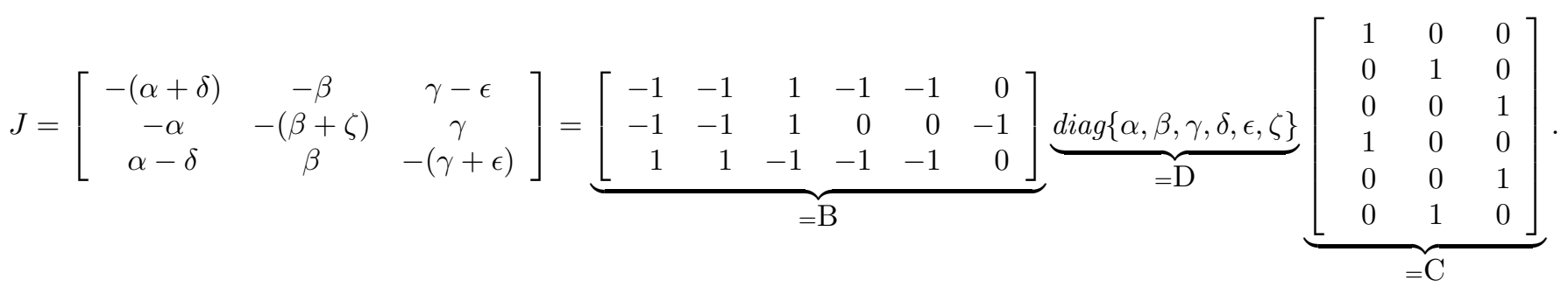

To compute the BDC-decomposition, an order must be chosen for the positive partial derivatives. Then, since the first derivative is $\alpha=\frac{\partial g_{a b}(a, b)}{\partial a}$, the first column of $B$ corresponds to $S_{1}$, associated with the reaction rate function $g_{a b}(\cdot, \cdot)$, and the first row of $C$ has a 1 entry in the first position, corresponding to variable a; and so on. Note that columns $S_{1}$ and $S_{3}$ are repeated twice in $B$ because $g_{a b}(\cdot, \cdot)$ and $g_{a c}(\cdot, \cdot)$ have two arguments.

Although the $B D C$-decomposition has an obvious connection with the system Jacobian, it is not just a local property, or a linearisation: based on a simple but powerful integral formula, it can be shown that the BDC-decomposition associated with a system is not only a local, but also a global property.

Generalising a result in [12, p. 108, Exercise 3.23], we can show the following.

Proposition 2 Given a continuously differentiable function $g(x), g: \mathcal{D} \subset \mathbb{R}^{n} \rightarrow \mathbb{R}^{n}$, where $\mathcal{D}$ is a convex domain with non-empty interior,

$$
g\left(x_{2}\right)-g\left(x_{1}\right)=\left(\int_{0}^{1} \frac{\partial g}{\partial x}\left(\sigma\left(x_{2}-x_{1}\right)+x_{1}\right) d \sigma\right)\left(x_{2}-x_{1}\right), \quad \forall x_{1}, x_{2} \in \mathcal{D} .
$$

Proof Denoting by $\varphi(\sigma) \doteq g\left(\sigma\left(x_{2}-x_{1}\right)+x_{1}\right)$ for $0 \leq \sigma \leq 1$, since

$$
\frac{d \varphi}{d \sigma}(\sigma)=\frac{\partial g}{\partial x}\left(\sigma\left(x_{2}-x_{1}\right)+x_{1}\right) \frac{d\left(\sigma\left(x_{2}-x_{1}\right)+x_{1}\right)}{d \sigma}=\frac{\partial g}{\partial x}\left(\sigma\left(x_{2}-x_{1}\right)+x_{1}\right)\left(x_{2}-x_{1}\right),
$$

we have

$$
g\left(x_{2}\right)-g\left(x_{1}\right)=\varphi(1)-\varphi(0)=\int_{0}^{1} \varphi^{\prime}(\sigma) d \sigma=\int_{0}^{1} \frac{\partial g}{\partial x}\left(\sigma\left(x_{2}-x_{1}\right)+x_{1}\right) d \sigma \quad\left(x_{2}-x_{1}\right)
$$

Consider system $(1)\left(\dot{x}(t)=S f(x(t))+f_{0}\right)$, with $x \in \mathcal{D}$ open and convex, along with the equilibrium condition $0=S f(\bar{x})+f_{0}$. Denoting $z \doteq x-\bar{x}$ and subtracting the two equations yields the shifted system

$$
\dot{z}=S[f(z+\bar{x})-f(\bar{x})]
$$


Since the system admits a $B D C$-decomposition, for any fixed equilibrium $\bar{x}$ we can consider $z=x-\bar{x}$ and the system can be equivalently rewritten (not linearised) as

$$
\dot{z}=[B \Delta(z) C] z
$$

In fact, an immediate application of the integral formula (3) to the right-hand side of system (4) provides

$$
\dot{z}=\left[\int_{0}^{1} J(\sigma z+\bar{x}) d \sigma\right] z
$$

In view of the local $B D C$-decomposition, equivalently

$$
\dot{z}=\left[B\left(\int_{0}^{1} D(\sigma z+\bar{x}) d \sigma\right) C\right] z=\left[B\left(\int_{0}^{1} \operatorname{diag}\left\{\frac{\partial f_{i}(\sigma z+\bar{x})}{\partial x_{j}}\right\} d \sigma\right) C\right] z .
$$

Therefore,

$$
\Delta(z)=\int_{0}^{1} D(\sigma z+\bar{x}) d \sigma=\int_{0}^{1} \operatorname{diag}\left\{\frac{\partial f_{i}(\sigma z+\bar{x})}{\partial x_{j}}\right\} d \sigma=\operatorname{diag}\left\{\int_{0}^{1} \frac{\partial f_{i}(\sigma z+\bar{x})}{\partial x_{j}} d \sigma\right\}=\operatorname{diag}\left\{\Gamma_{i j}(z)\right\}
$$

where $\Gamma_{i j}(z)=\int_{0}^{1} \frac{\partial f_{i}(\sigma z+\bar{x})}{\partial x_{j}} d \sigma$. Due to the monotonicity of the functions $f_{i}(\cdot)$, whose integral is computed on a non-zero interval, $\Gamma_{i j}(z)$ is strictly positive and admits a maximum and a minimum in any closed and bounded domain:

$$
0<\nu \leq \nu_{i j} \leq \Gamma_{i j}(z) \leq \mu_{i j} \leq \mu .
$$

Hence, a system admits a global $B D C$-representation (5) if and only if it admits a local $B D C$-decomposition (2).

Proposition 3 [6] Consider a nonlinear system (1) admitting equilibrium $\bar{x}$. The system can be equivalently written in the form $\dot{z}=B \Delta(z) C z$, where $z=x-\bar{x}$, if and only if it admits a BDC-decomposition, according to Definition 1.

Proof If $J(x)=B D(x) C$ for any $x$ in the domain, denoting $z=x-\bar{x}, J(z)=B D(z) C$ for any $z$. Then, integration exploiting the integral formula (3) entails the result, as discussed above. Conversely, if system (1) is equivalent to $\dot{z}=B \Delta(z) C z$, then linearisation immediately provides $J(z)=B D(z) C$, hence $J(x)=B D(x) C$.

\section{Influence analysis for biomolecular networks}

Given a dynamical system

$$
\begin{aligned}
\dot{x}(t) & =S f(x(t), u(t)) \\
y(t) & =\operatorname{Rh}(x(t))
\end{aligned}
$$

with state $x \in \mathbb{R}^{n}$, scalar input $u$ and scalar output $y$, suppose that the system is initially resting at the equilibrium $(\bar{x}, \bar{u})$, corresponding to the steady-state output $\bar{y}$, and then a persistent input perturbation $\bar{v}$ drives the system to the new equilibrium $(\bar{x}+\bar{z}, \bar{u}+\bar{v})$, corresponding to the new steady-state output $\bar{y}+\bar{w}$. The steady-state input-output influence is the sign of the output steady-state variation $\bar{w}$ due to the persistent positive input perturbation $\bar{v}$ :

$$
\frac{\operatorname{sign}[\bar{w}]}{\operatorname{sign}[\bar{v}]} \text {. }
$$

We call the influence structural (or structurally signed) if the sign does not depend on the value of the system parameters; the influence is sign-indeterminate otherwise.

The analysis of structurally signed input-output influences $[6,11]$ is relevant to many biological applications, and can provide interesting insight into the robust steady-state behaviour of systems both in ecology (see [8] and the discussion therein on the study of responses to press perturbation, a traditional approach to the study of ecological systems) and in biochemical and biomolecular systems (see $[7,9,10]$ ).

This section discusses in detail the steady-state input-output influence analysis for two examples of biomolecular networks, where the approach proposed in [3] reveals structurally signed influences that capture the inherent robustness of the system behaviour, regardless of parameter values. 
This extraordinary robustness, enabling a consistent signed behaviour in spite of parameter variations, is guaranteed by the system structure only, namely, by the "wiring" of the interactions among the interconnected species in the biological network (along with some qualitative properties of the individual dynamics, such as the monotonicity of the involved reaction rate functions).

The tool we introduce in [3] to capture the system structure and conduct a global influence analysis (without requiring any assumption on the magnitude of the input perturbation and without considering a linearised system approximation) is the global BDC-decomposition for systems with inputs and outputs. For the efficient computation of structurally signed steady-state input output influences, we employ the novel algorithm presented in [3]: to determine each input-output influence, the algorithm requires the computation of $2^{m-n}$ determinants, where $m$ is the number of unknown parameters and $n$ is the order of the dynamical system. In particular for large-scale systems, where $n$ is large and of the same order of magnitude of $m$, this is a significant reduction of the computational burden with respect to the vertex algorithm introduced for the first time in [11], which requires the computation of $2^{m}$ determinants.

Also, when the uncertain system parameters are known to belong to given intervals, based on the vertex algorithm proposed in [3], we can give non-conservative upper and lower bounds for the input-output steady-state sensitivity

$$
\frac{\bar{w}}{\bar{v}} .
$$

\subsection{An enzyme-driven biomolecular network}

We first consider the enzyme-driven biomolecular network described in [4, pp. 208-209]. In this biochemical reaction network, the enzyme $A$ catalyses the conversion of substrates $B$ and $C$ into the product $P$, by means of the formation of the intermediate complexes $D, E$ and $F$. The involved chemical reactions are the following:

$$
\begin{aligned}
& A+B \stackrel{g_{a b}}{\underset{g_{d}}{\longrightarrow}} D, A+C \stackrel{g_{a c}}{\underset{g_{e}}{\rightleftharpoons}} E, C+D \underset{g_{f}}{\stackrel{g_{c d}}{\rightleftharpoons}} F \underset{g_{b e}}{\stackrel{\hat{g}_{f}}{\rightleftharpoons}} B+E, F \stackrel{\tilde{g}_{f}}{\longrightarrow} A+P, \\
& \emptyset \stackrel{u_{b}}{\longrightarrow} B \stackrel{g_{b}}{\longrightarrow} \emptyset, \emptyset \stackrel{u_{c}}{\longrightarrow} C \stackrel{g_{c}}{\longrightarrow} \emptyset, P \stackrel{g_{p}}{\longrightarrow} \emptyset .
\end{aligned}
$$

We also consider an additional self-degradation $A \stackrel{g_{a}}{\longrightarrow} \emptyset$ and production $\emptyset \stackrel{u_{a}}{\longrightarrow} A$.

We denote species concentrations with the corresponding lowercase letter. Then, the associated system of differential equations, which describes the time evolution of species concentrations, is

$$
\begin{aligned}
\dot{a} & =-g_{a b}(a, b)+g_{d}(d)-g_{a c}(a, c)+g_{e}(e)+\tilde{g}_{f}(f)-g_{a}(a)+u_{a} \\
\dot{b} & =-g_{a b}(a, b)+g_{d}(d)-g_{b e}(b, e)+\hat{g}_{f}(f)-g_{b}(b)+u_{b} \\
\dot{c} & =-g_{a c}(a, c)+g_{e}(e)-g_{c d}(c, d)+g_{f}(f)-g_{c}(c)+u_{c} \\
\dot{d} & =g_{a b}(a, b)-g_{d}(d)-g_{c d}(c, d)+g_{f}(f) \\
\dot{e} & =g_{a c}(a, c)-g_{e}(e)-g_{b e}(b, e)+\hat{g}_{f}(f) \\
\dot{f} & =g_{b e}(b, e)+g_{c d}(c, d)-g_{f}(f)-\hat{g}_{f}(f)-\tilde{g}_{f}(f) \\
\dot{p} & =\tilde{g}_{f}(f)-g_{p}(p)
\end{aligned}
$$

If we take the $i$ th state variable as the system output, we can rewrite the system in the form (7)-(8), where $x=\left[\begin{array}{lllllll}a & b & c & d & e & f & p\end{array}\right]^{\top} \in \mathbb{R}^{7}$ (7 state variables) and $u=\left[\begin{array}{lll}u_{a} & u_{b} & u_{c}\end{array}\right]^{\top} \in \mathbb{R}^{3}$ (3 inputs), while matrix $S \in \mathbb{Z}^{7 \times 16}$ is

$$
S=\left[\begin{array}{cccccccccccccccc}
-1 & 1 & -1 & 1 & 1 & 0 & 0 & 0 & 0 & 0 & 0 & -1 & 0 & 1 & 0 & 0 \\
-1 & 1 & 0 & 0 & 0 & 1 & -1 & 0 & -1 & 0 & 0 & 0 & 0 & 0 & 1 & 0 \\
0 & 0 & -1 & 1 & 0 & 0 & 0 & -1 & 0 & 1 & -1 & 0 & 0 & 0 & 0 & 1 \\
1 & -1 & 0 & 0 & 0 & 0 & 0 & -1 & 0 & 1 & 0 & 0 & 0 & 0 & 0 & 0 \\
0 & 0 & 1 & -1 & 0 & 1 & 0 & 0 & -1 & 0 & 0 & 0 & 0 & 0 & 0 & 0 \\
0 & 0 & 0 & 0 & -1 & -1 & 0 & 1 & 1 & -1 & 0 & 0 & 0 & 0 & 0 & 0 \\
0 & 0 & 0 & 0 & 1 & 0 & 0 & 0 & 0 & 0 & 0 & 0 & -1 & 0 & 0 & 0
\end{array}\right]
$$

and vector $f(x, u)$ includes 13 reaction-rate functions, whose derivatives are all strictly positive in each argument, and 3 external inputs, $u_{a}, u_{b}$ and $u_{c}$ :

$$
f(x, u)=\left[g_{a b}(a, b) g_{d}(d) g_{a c}(a, c) g_{e}(e) \tilde{g}_{f}(f) \hat{g}_{f}(f) g_{b}(b) g_{c d}(c, d) g_{b e}(b, e) g_{f}(f) g_{c}(c) g_{a}(a) g_{p}(p) u_{a} u_{b} u_{c}\right]^{\top} .
$$


Moreover,

$$
R=V_{i}
$$

where $V_{i}$ denotes the row vector with 7 entries, whose $i$ th entry is 1 , while the others are zero, and

$$
h(x)=x .
$$

Therefore, we have $n=7$ state variables and $m=17$ unknown (but positive) parameters, corresponding to all the (nonzero) positive partial derivatives of the reaction-rate functions included in the vector function $f(x, u)$.

All the considered reaction-rate functions are assumed to be zero when at least one of the arguments is zero. Then, since $u_{a}>0, u_{b}>0$ and $u_{c}>0$, the unique equilibrium is positive. Indeed, at the equilibrium, from the first three equations $\bar{a}>0, \bar{b}>0$ and $\bar{c}>0$, from the fourth equation $\bar{d}>0$, from the fifth equation $\bar{e}>0$, from the sixth equation $\bar{f}>0$ and from the last equation $\bar{p}>0$. Hence, if this equilibrium is asymptotically stable, [3, Assumption 1] is satisfied, with $\mathcal{X}=\mathbb{R}_{\geq 0}^{7}$ and $\mathcal{U}=\mathbb{R}_{\geq 0}^{3}$.

This system admits a global $B D C$-decomposition, as discussed in [3]: matrix $B \in \mathbb{Z}^{7 \times 17}$ is

$$
B=\left[\begin{array}{ccccccccccccccccc}
-1 & -1 & 1 & -1 & -1 & 1 & 1 & 0 & 0 & 0 & 0 & 0 & 0 & 0 & 0 & -1 & 0 \\
-1 & -1 & 1 & 0 & 0 & 0 & 0 & 1 & -1 & 0 & -1 & -1 & 0 & 0 & 0 & 0 & 0 \\
0 & 0 & 0 & -1 & -1 & 1 & 0 & 0 & 0 & -1 & 0 & 0 & -1 & 1 & -1 & 0 & 0 \\
1 & 1 & -1 & 0 & 0 & 0 & 0 & 0 & 0 & -1 & 0 & 0 & -1 & 1 & 0 & 0 & 0 \\
0 & 0 & 0 & 1 & 1 & -1 & 0 & 1 & 0 & 0 & -1 & -1 & 0 & 0 & 0 & 0 & 0 \\
0 & 0 & 0 & 0 & 0 & 0 & -1 & -1 & 0 & 1 & 1 & 1 & 1 & -1 & 0 & 0 & 0 \\
0 & 0 & 0 & 0 & 0 & 0 & 1 & 0 & 0 & 0 & 0 & 0 & 0 & 0 & 0 & 0 & -1
\end{array}\right]
$$

the diagonal matrix $D_{x}$ includes 17 positive diagonal entries,

$$
D_{x}=\operatorname{diag}\{\alpha, \beta, \gamma, \delta, \epsilon, \zeta, \eta, \theta, \kappa, \lambda, \mu, \nu, \xi, \pi, \rho, \sigma, \tau\},
$$

corresponding to the partial derivatives $\alpha=\partial g_{a b} / \partial a, \beta=\partial g_{a b} / \partial b, \gamma=\partial g_{d} / \partial d, \delta=\partial g_{a c} / \partial a, \epsilon=\partial g_{a c} / \partial c, \zeta=\partial g_{e} / \partial e$, $\eta=\partial \tilde{g}_{f} / \partial f, \theta=\partial \hat{g}_{f} / \partial f, \kappa=\partial g_{b} / \partial b, \lambda=\partial g_{c d} / \partial c, \mu=\partial g_{b e} / \partial b, \nu=\partial g_{b e} / \partial e, \xi=\partial g_{c d} / \partial d, \pi=\partial g_{f} / \partial f, \rho=\partial g_{c} / \partial c$, $\sigma=\partial g_{a} / \partial a, \tau=\partial g_{p} / \partial p$, matrices $C \in \mathbb{Z}^{17 \times 7}$ and $E \in \mathbb{Z}^{7 \times 3}$ are

$$
C=\left[\begin{array}{lllllllllllllllll}
1 & 0 & 0 & 1 & 0 & 0 & 0 & 0 & 0 & 0 & 0 & 0 & 0 & 0 & 0 & 1 & 0 \\
0 & 1 & 0 & 0 & 0 & 0 & 0 & 0 & 1 & 0 & 1 & 0 & 0 & 0 & 0 & 0 & 0 \\
0 & 0 & 0 & 0 & 1 & 0 & 0 & 0 & 0 & 1 & 0 & 0 & 0 & 0 & 1 & 0 & 0 \\
0 & 0 & 1 & 0 & 0 & 0 & 0 & 0 & 0 & 0 & 0 & 0 & 1 & 0 & 0 & 0 & 0 \\
0 & 0 & 0 & 0 & 0 & 1 & 0 & 0 & 0 & 0 & 0 & 1 & 0 & 0 & 0 & 0 & 0 \\
0 & 0 & 0 & 0 & 0 & 0 & 1 & 1 & 0 & 0 & 0 & 0 & 0 & 1 & 0 & 0 & 0 \\
0 & 0 & 0 & 0 & 0 & 0 & 0 & 0 & 0 & 0 & 0 & 0 & 0 & 0 & 0 & 0 & 1
\end{array}\right]^{\top}, \quad E=\left[\begin{array}{lll}
1 & 0 & 0 \\
0 & 1 & 0 \\
0 & 0 & 1 \\
0 & 0 & 0 \\
0 & 0 & 0 \\
0 & 0 & 0 \\
0 & 0 & 0
\end{array}\right],
$$

matrices $D_{u}$ and $F$ are constant and both correspond to the $3 \times 3$ identity matrix, matrix $H$ is actually a 7-dimensional row vector, equal to $R=V_{i}$, while both $D_{y}$ and $L$ are $7 \times 7$ identity matrices.

The vertex algorithm reveals that $\psi(\Delta)=\operatorname{det}\left[-B \Delta_{x} C\right]>0$ structurally, for all possible $\Delta \succ 0$ : this is a necessary condition for stability of the system linearisation around the equilibrium point.

Since there are 3 inputs, we can consider each of them separately. If we consider input $u_{b}$ (hence, we take the second column of matrix $\left.E \Delta_{u} F\right)$ and output $y=x_{1}=a$, the corresponding expression for $\phi(\Delta)$ is

$$
\begin{aligned}
& \phi_{a b}(\Delta)=\operatorname{det}\left[\begin{array}{cc}
-B \Delta_{x} C & \left(-E \Delta_{u} F\right) \bullet 2 \\
H \Delta_{y} L & 0
\end{array}\right] \\
& =\operatorname{det}\left[\begin{array}{ccccccc|c}
-\hat{\alpha}-\hat{\delta}-\hat{\sigma} & -\hat{\beta} & -\hat{\epsilon} & \hat{\gamma} & \hat{\zeta} & \hat{\eta} & 0 & 0 \\
-\hat{\alpha} & -\hat{\beta}-\hat{\kappa}-\hat{\mu} & 0 & \hat{\gamma} & -\hat{\nu} & \hat{\theta} & 0 & 1 \\
-\hat{\delta} & 0 & -\hat{\epsilon}-\hat{\lambda}-\hat{\rho} & -\hat{\xi} & \hat{\zeta} & \hat{\pi} & 0 & 0 \\
\hat{\alpha} & \hat{\beta} & -\hat{\lambda} & -\hat{\gamma}-\hat{\xi} & 0 & \hat{\pi} & 0 & 0 \\
\hat{\delta} & -\hat{\mu} & \hat{\epsilon} & 0 & -\hat{\nu}-\hat{\zeta} & \hat{\theta} & 0 & 0 \\
0 & \hat{\mu} & \hat{\lambda} & \hat{\xi} & \hat{\nu} & -\hat{\eta}-\hat{\pi}-\hat{\theta} & 0 & 0 \\
0 & 0 & 0 & 0 & 0 & \hat{\eta} & -\hat{\tau} & 0 \\
\hline 1 & 0 & 0 & 0 & 0 & 0 & 0 & 0
\end{array}\right],
\end{aligned}
$$


where the diagonal entries of $\Delta_{x}$ (the Greek letters with the hat) represent the integral of the positive parameters in $D_{x}$, as discussed in [3].

If we consider all possible input-output pairs (including additive inputs to all the state equations, not only $u_{a}, u_{b}$ and $u_{c}$ ), the steady-state influence matrix is

$$
\Sigma=\left[\begin{array}{ccccccc}
+ & 0 & 0 & + & + & + & 0 \\
- & + & - & ? & - & ? & 0 \\
- & - & + & - & ? & ? & 0 \\
? & + & ? & ? & ? & ? & 0 \\
? & ? & + & ? & ? & ? & 0 \\
+ & + & + & + & + & + & 0 \\
+ & + & + & + & + & + & +
\end{array}\right]
$$

where columns $\Sigma_{\bullet 1}, \Sigma_{\bullet 2}$ and $\Sigma_{\bullet 3}$ show the influences on each state component, taken as the system output, due to $u_{a}, u_{b}$ and $u_{c}$, respectively. Interestingly, 35 out of 49 entries of the influence matrix are structurally signed.

There are 8 influence matrix entries equal to zero. The test proposed in [3] allows us to understand, for each of these entries, if this is due to perfect adaptation, or if the transfer function is identically zero (due to a lack of either reachability or observability). In particular, computing the numerator of the transfer function

$$
\phi(\Delta, s)=\operatorname{det}\left[\begin{array}{cc}
s I-B \Delta_{x} C & \left(-E \Delta_{u} F\right) \bullet j \\
H \Delta_{y} L & 0
\end{array}\right]
$$

(where $j$ depends on the choice of the input) for 7 different nonzero values of $s$ enables us to check that $\phi(\Delta, s)$ is identically zero for the first six entries of column $\Sigma_{\bullet}$ (indeed, the variables $a$ to $f$ are not reachable by an additive input applied to the equation of $p$ ), while it is not identically zero for entries $\Sigma_{12}$ and $\Sigma_{13}$, corresponding to actual perfect adaptation.

The novel algorithm we have employed provides a global result for influence analysis and significantly reduces the computational complexity: to structurally assess each of the signed influences, it has been enough to compute $2^{m-n}=2^{17-7}=2^{10}=1024$ determinants, instead of $2^{m}=2^{17}=131072$ (as required by the vertex algorithm in [11])

With the methods introduced in [3], we can also perform an input-output sensitivity analysis based on an efficient vertex algorithm. When the uncertain diagonal entries $\Delta_{k}$ of matrix $\Delta$ are bounded as $1 \leq \Delta_{k} \leq 10$, the algorithm provides the following lower and upper bounds for the steady-state input-output sensitivities:

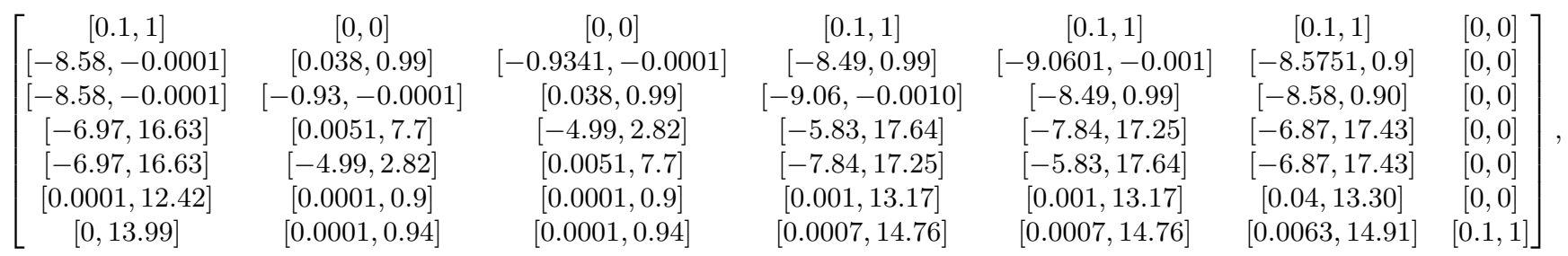

which are fully consistent with the signed influence matrix $\Sigma$. In fact, the lower and upper bounds have a different sign only when the corresponding entry of the steady-state influence matrix is $\Sigma_{i j}=$ ?.

\subsection{Individual mRNA-protein dynamics in a multicellular network}

In this section, we consider the synthetic multicellular network of repressilators coupled by quorum sensing that is discussed in [5]. Each repressilator involves three genes $\left(X_{1}, X_{2}\right.$ and $\left.X_{3}\right)$, each expressing a protein $\left(X_{4}, X_{5}\right.$ and $X_{6}$ respectively) that inhibits the transcription of another gene, in a negative feedback loop. The coupling through quorum sensing is enabled by cell-to-cell communication based on the autoinducer $\left(X_{7}\right.$, a small molecule able to diffuse through the cell membrane). The autoinducer is synthesised due to the action of protein $X_{4}$, and in turn activates the transcription of gene $X_{3}$.

The dynamics of mRNA and protein evolution in each of the cells is therefore described by the system of ordinary 
differential equations [5], [4, pp. 314-315]:

$$
\begin{aligned}
& \dot{x}_{1}=-\alpha x_{1}+g_{6}\left(x_{6}\right) \\
& \dot{x}_{2}=-\beta x_{2}+g_{4}\left(x_{4}\right) \\
& \dot{x}_{3}=-\gamma x_{3}+g_{5}\left(x_{5}\right)+g_{7}\left(x_{7}\right) \\
& \dot{x}_{4}=-\delta x_{4}+\pi x_{1} \\
& \dot{x}_{5}=-\varepsilon x_{5}+\rho x_{2} \\
& \dot{x}_{6}=-\phi x_{6}+\sigma x_{3} \\
& \dot{x}_{7}=\tau x_{4}-\omega x_{7}+\eta\left(u_{7}-x_{7}\right)
\end{aligned}
$$

where $g_{4}, g_{5}, g_{6}$ are decreasing Hill-type functions,

$$
g_{4}\left(x_{4}\right)=\frac{\kappa_{p, 4}}{\kappa_{a, 4}+x_{4}^{\kappa_{H, 4}}}, \quad g_{5}\left(x_{5}\right)=\frac{\kappa_{p, 5}}{\kappa_{a, 5}+x_{5}^{\kappa_{H, 5}}}, \quad g_{6}\left(x_{6}\right)=\frac{\kappa_{p, 6}}{\kappa_{a, 6}+x_{6}^{\kappa_{H, 6}}},
$$

$g_{7}$ is an increasing Michaelis-Menten function,

$$
g_{7}\left(x_{7}\right)=\frac{\kappa_{p, 7} x_{7}}{\kappa_{a, 7}+x_{7}}
$$

and $u_{7}$ denotes the average extracellular concentration of the autoinducer.

If we take the $i$ th state variable as the system output, we can rewrite the system in the form (7)-(8), where $x=\left[\begin{array}{lllllll}x_{1} & x_{2} & x_{3} & x_{4} & x_{5} & x_{6} & x_{7}\end{array}\right]^{\top} \in \mathbb{R}^{7}$ (7 state variables) and $u=u_{7} \in \mathbb{R}$ (a single input), while matrix $S \in \mathbb{Z}^{7 \times 17}$ is

$$
S=\left[\begin{array}{ccccccccccccccccc}
-1 & 0 & 0 & 0 & 0 & 0 & 0 & 0 & -1 & 0 & 0 & 0 & 0 & 0 & 0 & 0 & 0 \\
0 & -1 & 0 & 0 & 0 & 0 & 0 & 0 & 0 & -1 & 0 & 0 & 0 & 0 & 0 & 0 & 0 \\
0 & 0 & -1 & 0 & 0 & 0 & 0 & 0 & 0 & 0 & -1 & 1 & 0 & 0 & 0 & 0 & 0 \\
0 & 0 & 0 & -1 & 0 & 0 & 0 & 0 & 0 & 0 & 0 & 0 & 1 & 0 & 0 & 0 & 0 \\
0 & 0 & 0 & 0 & -1 & 0 & 0 & 0 & 0 & 0 & 0 & 0 & 0 & 1 & 0 & 0 & 0 \\
0 & 0 & 0 & 0 & 0 & -1 & 0 & 0 & 0 & 0 & 0 & 0 & 0 & 0 & 1 & 0 & 0 \\
0 & 0 & 0 & 0 & 0 & 0 & -1 & -1 & 0 & 0 & 0 & 0 & 0 & 0 & 0 & 1 & 1
\end{array}\right]
$$

and vector $f(x, u)$ includes 16 reaction-rate functions, whose derivatives are either strictly positive or strictly negative in each argument, and 1 external input, $u_{7}$ :

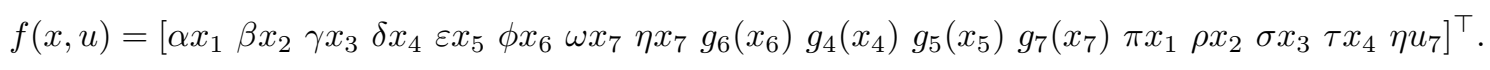

Also,

$$
R=V_{i}
$$

where $V_{i}$ denotes the row vector with 7 entries, whose $i$ th entry is 1 , while the others are zero, and

$$
h(x)=x .
$$

Therefore, the system has $n=7$ state variables and $m=16$ unknown (but positive) parameters, corresponding to the absolute values of all the (nonzero) partial derivatives of the functions included in $f(x, u)$.

Since $u_{7}>0$, the unique equilibrium is positive. Indeed, at the equilibrium, from the last equation it must be $\bar{x}_{7}>0$; then from the third equation $\bar{x}_{3}>0$, from the sixth equation $\bar{x}_{6}>0$, from the first equation $\bar{x}_{1}>0$, from the fourth equation $\bar{x}_{4}>0$, from the second equation $\bar{x}_{2}>0$ and from the fifth equation $\bar{x}_{5}>0$. Hence, under asymptotic stability assumptions, [3, Assumption 1] is satisfied, with $\mathcal{X}=\mathbb{R}_{\geq 0}^{7}$ and $\mathcal{U}=\mathbb{R}_{\geq 0}$.

The system admits a global $B D C$-decomposition where matrix $B \in \mathbb{Z}^{7 \times 16}$ is

$$
B=\left[\begin{array}{cccccccccccccccc}
-1 & 0 & 0 & 0 & 0 & 0 & 0 & 0 & -1 & 0 & 0 & 0 & 0 & 0 & 0 & 0 \\
0 & -1 & 0 & 0 & 0 & 0 & 0 & 0 & 0 & -1 & 0 & 0 & 0 & 0 & 0 & 0 \\
0 & 0 & -1 & 0 & 0 & 0 & 0 & 0 & 0 & 0 & -1 & 1 & 0 & 0 & 0 & 0 \\
0 & 0 & 0 & -1 & 0 & 0 & 0 & 0 & 0 & 0 & 0 & 0 & 1 & 0 & 0 & 0 \\
0 & 0 & 0 & 0 & -1 & 0 & 0 & 0 & 0 & 0 & 0 & 0 & 0 & 1 & 0 & 0 \\
0 & 0 & 0 & 0 & 0 & -1 & 0 & 0 & 0 & 0 & 0 & 0 & 0 & 0 & 1 & 0 \\
0 & 0 & 0 & 0 & 0 & 0 & -1 & -1 & 0 & 0 & 0 & 0 & 0 & 0 & 0 & 1
\end{array}\right]
$$


the diagonal matrix $D_{x}$ includes 16 positive diagonal entries,

$$
D_{x}=\{\alpha, \beta, \gamma, \delta, \varepsilon, \phi, \omega, \eta, \kappa, \lambda, \mu, \nu, \pi, \rho, \sigma, \tau\} \succ 0,
$$

corresponding to the absolute values of the partial derivatives, where $\kappa=-\partial g_{6} / \partial x_{6}>0, \lambda=-\partial g_{4} / \partial x_{4}>0$, $\mu=-\partial g_{5} / \partial x_{5}>0, \nu=\partial g_{7} / \partial x_{7}>0$, matrix $C \in \mathbb{Z}^{16 \times 7}$ is

$$
C=\left[\begin{array}{llllllllllllllll}
1 & 0 & 0 & 0 & 0 & 0 & 0 & 0 & 0 & 0 & 0 & 0 & 1 & 0 & 0 & 0 \\
0 & 1 & 0 & 0 & 0 & 0 & 0 & 0 & 0 & 0 & 0 & 0 & 0 & 1 & 0 & 0 \\
0 & 0 & 1 & 0 & 0 & 0 & 0 & 0 & 0 & 0 & 0 & 0 & 0 & 0 & 1 & 0 \\
0 & 0 & 0 & 1 & 0 & 0 & 0 & 0 & 0 & 1 & 0 & 0 & 0 & 0 & 0 & 1 \\
0 & 0 & 0 & 0 & 1 & 0 & 0 & 0 & 0 & 0 & 1 & 0 & 0 & 0 & 0 & 0 \\
0 & 0 & 0 & 0 & 0 & 1 & 0 & 0 & 1 & 0 & 0 & 0 & 0 & 0 & 0 & 0 \\
0 & 0 & 0 & 0 & 0 & 0 & 1 & 1 & 0 & 0 & 0 & 1 & 0 & 0 & 0 & 0
\end{array}\right]^{\top}
$$

matrix $E$ is actually a 7-dimensional column vector

$$
E=\left[\begin{array}{lllllll}
0 & 0 & 0 & 0 & 0 & 0 & 1
\end{array}\right]^{\top},
$$

matrix $D_{u}$ and matrix $F$ are actually scalars, $D_{u}=\eta$ and $F=1$, matrix $H$ is actually a 7-dimensional row vector, equal to $R=V_{i}$, while both $D_{y}$ and $L$ are $7 \times 7$ identity matrices.

The proposed algorithm reveals that, also in this case, $\psi(\Delta)=\operatorname{det}\left[-B \Delta_{x} C\right]>0$ structurally, for all possible $\Delta_{x} \succ 0$ (which is necessary for the linearised system to be stable).

If we consider output $y=x_{3}$, the corresponding expression for $\phi(\Delta)$ is

$$
\phi_{37}(\Delta)=\operatorname{det}\left[\begin{array}{cc}
-B \Delta_{x} C & -E \Delta_{u} F \\
H \Delta_{y} L & 0
\end{array}\right]=\operatorname{det}\left[\begin{array}{ccccccc|c}
-\hat{\alpha} & 0 & 0 & 0 & 0 & -\hat{\kappa} & 0 & 0 \\
0 & -\hat{\beta} & 0 & -\hat{\lambda} & 0 & 0 & 0 & 0 \\
0 & 0 & -\hat{\gamma} & 0 & -\hat{\mu} & 0 & \hat{\nu} & 0 \\
\hat{\pi} & 0 & 0 & -\hat{\delta} & 0 & 0 & 0 & 0 \\
0 & \hat{\rho} & 0 & 0 & -\hat{\epsilon} & 0 & 0 & 0 \\
0 & 0 & \hat{\sigma} & 0 & 0 & -\hat{\phi} & 0 & 0 \\
0 & 0 & 0 & \hat{\tau} & 0 & 0 & -\hat{\omega}-\hat{\eta} & \hat{\eta} \\
\hline 0 & 0 & 1 & 0 & 0 & 0 & 0 & 0
\end{array}\right],
$$

where the diagonal entries of $\Delta_{x}$ (the Greek letters with the hat) represent the integral of the positive parameters in $D_{x}$, as discussed in [3].

We can consider all possible input-output pairs (including additive inputs to all the state equations, not only $u_{7}$ ). Then, the steady-state influence matrix is

$$
\Sigma=\left[\begin{array}{ccccccc}
+ & + & - & - & + & - & - \\
- & + & + & - & - & + & + \\
+ & - & + & + & - & - & + \\
+ & + & - & + & + & - & - \\
- & + & + & - & + & + & + \\
+ & - & + & + & - & + & + \\
+ & + & - & + & + & - & +
\end{array}\right],
$$

where column $\Sigma_{\bullet}$ shows the influence on each state component, taken as the system output, due to input $u_{7}$.

Quite surprisingly, all the entries of the influence matrix are structurally signed (either positive or negative), regardless of the chosen parameters.

The influence analysis results achieved are global. Also, to assess the structural sign of each input-output influence, the new algorithm has required to compute $2^{m-n}=2^{16-7}=2^{9}=512$ determinants, instead of $2^{m}=2^{16}=65536($ as required by the vertex algorithm in [11]). Thus, the computational complexity is significantly reduced.

An efficient vertex algorithm allows us to perform a robust input-output sensitivity analysis, when the uncertain diagonal entries of matrix $\Delta$ are subject to known upper and lower bounds. In particular, if $1 \leq \Delta_{k} \leq 10$, the 
algorithm provides the following lower and upper bounds for the steady-state input-output sensitivities:

$$
\left[\begin{array}{ccccccc}
{[0,1]} & {[0,9.99]} & {[-62.5,-0.0001]} & {[-1,0]} & {[0.0001,86.96]} & {[-9.94,0]} & {[-9.78,0]} \\
{[-90.50,0]} & {[0.0001,1]} & {[0,9.43]} & {[-9.99,0]} & {[-1,0]} & {[0.0001,86.96]} & {[0,8.32]} \\
{[0.0001,9.99]} & {[-90.91,0]} & {[0,1]} & {[0.0004,90.98]} & {[-9.99,0]} & {[-1,0]} & {[0,4.98]} \\
{[0,9.94]} & {[0,1]} & {[-16.39,-0.0001]} & {[0,1]} & {[0,9.43]} & {[-62.5,-0.0001]} & {[-1,0]} \\
{[-90.83,0]} & {[0.0005,9.99]} & {[0,1]} & {[-90.91,0]} & {[0.0001,1]} & {[0,9.99]} & {[0,4.76]} \\
{[0,1]} & {[-90.83,0]} & {[0,9.94]} & {[0.0001,9.99]} & {[-90.5,0]} & {[0,1]} & {[0,33.3]} \\
{[0,33.3]} & {[0,4.76]} & {[-1,0]} & {[0,4.98]} & {[0,8.32]} & {[-9.78,0]} & {[0.0001,0.5]}
\end{array}\right] .
$$

The achieved upper and lower bounds are fully consistent with the signed influence matrix $\Sigma$ : in fact, lower and upper bounds for each entry never have a different sign, consistently with the fact that $\Sigma$ is fully sign-definite.

\section{References}

[1] F. Blanchini and G. Giordano, "Piecewise-linear Lyapunov functions for structural stability of biochemical networks", Automatica, 50(10):2482-2493, 2014.

[2] F. Blanchini and G. Giordano, "Polyhedral Lyapunov functions structurally ensure global asymptotic stability of dynamical networks iff the Jacobian is non-singular", Automatica, 86(12):183-191, 2017.

[3] F. Blanchini and G. Giordano, "BDC-decomposition for global influence analysis", submitted to IEEE Control Systems Letters, 2018.

[4] L. Chen, R. Wang, C. Li, and K. Aihara, Modeling Biomolecular Networks in Cells, Springer, 2005.

[5] J. Garcia-Ojalvo, M. Elowitz, and S. H. Strogatz, "Modeling a synthetic multicellular clock: Repressilators coupled by quorum sensing", Proc Natl Acad Sci U.S.A., 101:10955-10960, 2004.

[6] G. Giordano, Structural Analysis and Control of Dynamical Networks, Ph.D. thesis, University of Udine, Italy, 2016.

[7] G. Giordano, "CERT-mediated ceramide transfer is a structurally tunable flow-inducing mechanism with structural feed-forward loops", Royal Society Open Science, in press, 2018.

[8] G. Giordano, C. Altafini, "Qualitative and quantitative responses to press perturbations in ecological networks", Scientific Reports, 7:11378, 2017.

[9] G. Giordano, C. Altafini, "Interaction sign patterns in biological networks: from qualitative to quantitative criteria", IEEE Conference on Decision and Control, pp. 5348-5353, 2017.

[10] G. Giordano, E. Franco, "Negative feedback enables structurally signed steady-state influences in artificial biomolecular networks", IEEE Conference on Decision and Control, pp. 3369-3374, 2016.

[11] G. Giordano, C. Cuba Samaniego, E. Franco, F. Blanchini, "Computing the structural influence matrix for biological systems", Journal of Mathematical Biology, 72(7):1927-1958, 2016.

[12] H. K. Khalil, Nonlinear systems. Prentice Hall, 2002. 